\title{
Polyaniline promotes peripheral nerve regeneration by enhancement of the brain-derived neurotrophic factor and ciliary neurotrophic factor expression and activation of the ERK1/2/MAPK signaling pathway
}

\author{
LIN FAN $^{1}$, YAN XIONG $^{1}$, ZHEN FU $^{2}$, DINGFENG XU ${ }^{3}$, LEI WANG ${ }^{1}$, YONG CHEN $^{4}$, HAOYANG XIA $^{1}$, \\ NA PENG ${ }^{1}$, SHAOJUN YE $^{1}$, YANFENG WANG ${ }^{1}$, LINA ZHANG $^{3}$ and QIFA YE ${ }^{1,2}$ \\ ${ }^{1}$ Zhongnan Hospital of Wuhan University, Institute of Hepatobiliary Diseases of Wuhan University, Transplant \\ Center of Wuhan University, Hubei Key Laboratory of Medical Technology on Transplantation, Wuhan, \\ Hubei 430071; ${ }^{2}$ Research Center of National Health Ministry on Transplantation Medicine Engineering and \\ Technology, The 3rd Xiangya Hospital of Central South University, Changsha, Hunan 410013; ${ }^{3}$ College \\ of Chemistry \& Molecule Sciences, Wuhan University, Wuhan, Hubei 430081; ${ }^{4}$ Department of Abdominal \\ Laparoscopic Surgery, Qinghai University Affiliated Hospital, Xining, Qinghai 810001, P.R. China
}

Received January 13, 2017; Accepted August 2, 2017

DOI: $10.3892 / \mathrm{mmr} .2017 .7534$

\begin{abstract}
A previous study has demonstrated a progression in the nerve regeneration by polyaniline/cellulose (PANI/RC), although the underlying mechanism was not elucidated. In the present study, regenerated nerves were investigated, using histological techniques, functional assays and western blot analysis. The triceps surae muscle weight ratio percentages of the sham, regenerated cellulose (RC) and the PANI/RC groups were $38.88 \pm 4.76$ and $76.32 \pm 7.11 \%$, respectively. The thickness of the myelin sheath for the aforementioned groups were as follows: $1.2 \pm 0.27 ; 0.49 \pm 0.21$ and $0.93 \pm 0.28 \mu$ l. Western blot analysis demonstrated that the ciliary neurotrophic factor (CNTF) and brain-derived neurotrophic factor (BDNF) were highly expressed in the regenerated nerve in the presence of polyaniline. Phosphorylated extracellular kinase (p-ERK)1/2 expression in the PANI/RC group was significantly elevated compared with the $\mathrm{RC}$ group (1.83-fold) and the sham group (4.92-fold). The expression of the axon sprout-associated
\end{abstract}

Correspondence to: Dr Qifa Ye, Zhongnan Hospital of Wuhan University, Institute of Hepatobiliary Diseases of Wuhan University, Transplant Center of Wuhan University, Hubei Key Laboratory of Medical Technology on Transplantation, Wuhan University, 169 East Lake Road, Wuchang, Wuhan, Hubei 430071, P.R. China E-mail: yqf_china@163.com

Professor Lina Zhang, College of Chemistry \& Molecule Sciences, Wuhan University, 299 Bayi Road, Wuhan, Hubei 430081, P.R. China

E-mail: zhangln@whu.edu.cn

Key words: polyaniline, sciatic nerve, regeneration, Schwann cells, brain-derived neurotrophic factor, ciliary neurotrophic factor proteins, such as Tau, $\alpha$-tubulin and growth associated protein-43, were increased $(1.64,1.59$ and 1.24-fold, respectively) compared with the RC group. The results demonstrated that PANI enhances the expression and secretion of BDNF and CNTF, activates the ERK1/2 signaling pathway and increases the expression levels of the GAP-43, Tau and $\alpha$-tubulin, suggesting an insight into nerve regeneration and possible clinical interventions in nerve injury.

\section{Introduction}

The incidence of new onset peripheral nerve injury is increasing worldwide. More than one million cases with new onset peripheral nerve injury is presented annually worldwide (1). The peripheral nerve injuries can result from trauma, cancer or congenital defects. Regarding the clinical and basic research, the sciatic nerve injury and regeneration process has been a focus of major investigation in medicine. The nerve fiber growth velocity is very slow and is estimated to $1-2 \mathrm{~mm} /$ day in humans $(2,3)$. The autologous nerve graft is considered the best treatment option, although significant drawbacks are encountered, such as the limited donor source, the donor site morbidity, the multiple surgical sites and the possible size mismatch (4). Consequently, novel methods to replace the autologous nerve graft for nerve gap reconstruction are continuously evaluated.

In the previous study conducted by our group, the RSC96 cell line was used to evaluate the biological applications of the polyaniline/regenerated cellulose (PANI/RC) that enhanced RSC96 cell adhesion and proliferation (5). Scanning electron microscopy demonstrated the adhesion of RSC96 cells via pseudopodium extension on the PANI sub-micrometer dendritic particles (5). It is well established that Schwann cells serve a critical role in the peripheral nerve regeneration (6). In the present study, it was hypothesized that PANI is involved 
in the latter process via the regulation of Schwann cells. The specific function of PANI in nerve regeneration was detected at the molecular level. During the aforementioned process, the involvement of a complex interlinked cell signaling pathway was identified. The recruitment of cytokines and neurotrophins at the site of the lesion resulted in the activation of a series of molecular and cellular signaling pathways involved in neuronal and non-neuronal cell communication (7,8). Such mechanisms may include the generation of new neurons, glia, axons, myelin or synapses that in turn promote nerve regeneration (8).

Neurotrophins are proteins that help stimulate and control neurogenesis. Brain derived neurotrophic factor (BDNF) is one of the most active neurotrophins (9) that supports the survival of existing neurons and promotes the growth and differentiation of new neurons and synapses $(10,11)$. In addition, the application of exogenous BDNF to the nerve topical lesion has been reported to potentiate axon regrowth and maturation during peripheral nerve regeneration (12).

Cytokine ciliary neurotrophic factor (CNTF) is an additional neurotrophin that is required for nerve regeneration (13). CNTF is secreted by Schwann cells and astrocytes, and the application of exogenous CNTF to the nerve lesion has also been reported to promote axon regrowth and maturation during peripheral nerve regeneration (14-17). However, it remains unclear whether the role of endogenous CNTF in the activation of cell growth and differentiation is associated with PANI, following peripheral nerve injury.

In the present study, the ability of PANI/RC to promote rat sciatic nerve regeneration was demonstrated by monitoring the morphological and molecular changes of the regenerated nerve fibers using histological, immunohistochemical and western blotting techniques.

\section{Materials and methods}

Preparation of PANI/RC conduit and cellulose conduit. The PANI/RC hydrogels were prepared through the interfacial polymerization via a $U$ tube, of which a cellulose hydrogel was sandwiched in the middle as previous described (5). The PANI/RC and RC hydrogels were rolled to fabricate PANI/RC and RC conduits. The final length of conduits was $12 \mathrm{~mm}$, with an inner diameter of $1.5 \mathrm{~mm}$ and a tube wall thickness of $1 \mathrm{~mm}$.

Animals. Adult male Sprague-Dawley rats, (age, 8-10 weeks, weight, $210 \pm 10 \mathrm{~g}, \mathrm{n}=45$ ) were obtained from the Hubei Provincial Center for Disease Control and Prevention in Wuhan, China. The experimental procedures involving animals were carried out in accordance with National Institutes of Health (Bethesda, MD, USA) guide for the care and use of laboratory animals and The Code of Ethics of the World Medical Association. The study received ethics approval from the Ethics Committee of Zhongnan Hospital of Wuhan University (Wuhan, China).

Surgical procedure. The animals were divided into three groups (15 rats for each group): Sham (the sciatic nerve was exposed in the absence of nerve tissue disposal), RC (the defected sciatic nerve was connected with the RC conduit) and
PANI/RC (the defected sciatic nerve was connected with the $\mathrm{PANI} / \mathrm{RC}$ conduit). The $5 \mathrm{~mm}$ defects in the sciatic nerve were produced by removing the nerve tissue which was repaired with the nerve conduits as reported previously (18-20).

Triceps weight analysis. The triceps surae muscles from the experimental and the contra lateral sides (non-operated) were weighed in order to estimate the relative weight ratio 3 months following surgery, according to the following formula:

$$
\text { triceps weight } \%=\frac{\text { triceps weight of the operated leg }}{\text { triceps weight of the unoperated leg }} \times 100 \%
$$

Histological assessment. The harvested nerve grafts were fixed in a cold buffered $4 \%$ paraformaldehyde solution or cold buffered $3 \%$ glutaraldehyde solution. Following fixation, the former part of tissues in each group were embedded in olefin, cut in $4 \mathrm{~mm}$ slices and stained with hematoxylin (10 min at room temperature) and eosin ( $2 \mathrm{~min}$ at room temperature; H\&E) or toluidine blue ( $3 \mathrm{~min}$ at room temperature). The latter part of the nerve sections were fixed in a cold buffered $3 \%$ glutaraldehyde solution for transmission electron microscopy TEM examination (JEM-1200 EX, JEOL Ltd., Tokyo, Japan). A total of 200 nerve fibers in 10 random areas were selected by two pathologists for the fiber size analysis. All nerve sections were observed under a light microscope (TE2000-U, Nikon Corporation, Tokyo, Japan). An image analysis system (Image-Pro Plus Version6.0, Media Cybernetics Inc., Rockville, MD, USA) was used to determine the number and areas of the individual myelinated axons.

Immunohistochemical evaluation. The expression of the extracellular signal-regulated kinase (ERK) 1/2/ mitogen-associated protein kinase (MAPK) was evaluated in nerve paraffin sections of the regenerated nerve tissues via immunohistochemical analysis. After being blocked (10 min at room temperature) using 5\% sham goat serum (cat. no. AR1009; Wuhan Boster Biological Technology Ltd., Wuhan, China), the sections were incubated with a rabbit anti-rat ERK1/2 antibody (cat. no. 16443-1-AP; 1:50; ProteinTech Group, Inc., Wuhan, China) or MAPK (cat. no. 9212; 1:100; Cell Signaling Technology Ltd., Danvers, MA, USA) at $4^{\circ} \mathrm{C}$ overnight and then with biotinylated secondary antibodies (cat. no. AB501-01A; 1:500; SinoBiological Inc., Shanghai, China) for $20 \mathrm{~min}$ at $37^{\circ} \mathrm{C}$. The sections were reacted with a Strept Avidin Biotin Complex (100 $\mu \mathrm{l}$; cat. no. PV-9000; Zhongshan Gold Bridge Biological Technology Ltd., Beijing, China) for $20 \mathrm{~min}$ at room temperature and finally stained with 3,3-Diaminobenzidine (cat. no. AR1022; Wuhan Boster Biological Technology Ltd., Wuhan, China). The positive ratios (200 cells in 10 random areas counted) of ERK1/2(MAPK) were observed by microscope (TE2000-U, Nikon Corporation, Tokyo, Japan) and analyzed by an image analysis system (Image-Pro Plus version 6.0; Media Cybernetics Inc., Rockville, MD, USA).

Western blot analysis. The total protein content was extracted from regenerated or sham nerve in a lysis buffer (cat. no. P0013B; Beyotime Biotechnology Ltd., Shanghai, China) 
containing $50 \mathrm{mM}$ Tris $\mathrm{HCl}, 1 \% \mathrm{NP}-40,150 \mathrm{mM} \mathrm{NaCl}, 0.1 \%$ SDS, $1 \mathrm{mM}$ PMSF and $1 \mathrm{mM} \mathrm{Na}_{3} \mathrm{VO}_{4}$. The protein concentration of the samples was determined by the Bicinchoninic Acid assay kit. Bovine serum albumin (5\%; cat. no. 164210-100; Procell Life Science \& Technology Co., Ltd., Wuhan, China) was used as a standard. An equal amount $(30 \mu \mathrm{g})$ of protein was loaded on polyacrylamide gels containing 10-12\% SDS and transferred to polyvinylidene fluoride membranes. The membranes were probed with specific antibodies against nerve growth factor (cat. no. BA0611-2; 1:200; Wuhan Boster Biotechnology Co., Ltd); tau (cat. no. ab32057; 1:5,000; Abcam, Cambridge, MA, USA); S100 (cat. no. ab52642; 1:5,000; Abcam); growth-associated protein-43 (GAP-43; cat. no. ab75810; 1:5,000; Abcam); $\alpha$-tubulin (cat. no. ab15246; 1:500; Abcam), BDNF (cat. no. SC-546; 1:300; Santa Cruz Biotechnology, Inc., Dallas, TX, USA), CNTF (cat. no. ab46172; 1:3,000; Abcam); ERK (cat. no. 16443-1-AP; 1:1,000; ProteinTech Group, Inc.); phosphorylated (p)-ERK1/2 (cat. no. 4370; 1:2,000; Cell Signaling Technology Inc.); $\beta$-actin (cat. no. BM0627; 1:200; Wuhan Boster Biotechnology Co., Ltd.) and GAPDH (cat. no. AB-P-R 001; 1:1,000; Hangzhou Goodhere Biotechnology Co., Ltd., Hangzhou, China). GAPDH and $\beta$-actin were used as internal controls. The protein detection was carried out using the enhanced chemiluminescence detection system (cat. no. NCI5079; Thermo Fisher Scientific, Inc., Waltham, MA, USA) and the membranes were exposed on Kodak X-Omat films. The protein levels were semi quantified by densitometric analysis of the integrated area (pixels) of the protein bands using the BandScan software version 4.3 (http://download1.bio1000. $\mathrm{com} / \mathrm{soft} / \mathrm{biology} /$ BandScan5.0.rar). The numerical values for the protein band intensities were produced following normalization of the target protein expression with the expression value corresponding to the GAPDH or $\beta$-actin band.

Statistical analysis. SPSS 21.0 statistical software was used for the statistical analysis of the relevant data (IBM Corp., Armonk, NY, USA). The data are expressed as the mean \pm standard deviation. The differences between the two groups were compared using the student t-test. The differences among several groups were analyzed using one-way analysis of variance followed by least significance difference multiple-comparison tests. $\mathrm{P}<0.05$ was considered to indicate a statistically significant difference.

\section{Results}

General post-operative observations. The conduits for nerve reconstruction are indicated in the nerve condition intra-operatively (Fig. 1A and B) and 3 months post-operation (Fig. 1C and D). Fig. 1 indicates the sciatic nerve reconstruction that was conducted using the following materials: PANI/RC (Fig. 1A and C) and RC (Fig. 1B and D). The PANI/RC conduit indicated favorable biocompatibility performance (Fig. 1C), while the RC conduit was wrapped with thick fibrous connective tissue (Fig. 1D).

Triceps weight analysis. Nerve recovery is indirectly reflected by muscle recovery. The triceps surae muscle weight ratio is estimated by the formula: Operation side/contralateral side x100\%. Sham group: $99.70 \pm 2.29$; RC group: $38.88 \pm 4.76$; PANI/RC group: $76.32 \pm 7.11, \mathrm{P}<0.01$ (Fig. 1E). In terms of triceps surae muscle weight ratio, the PANI/RC group demonstrated better motor function recovery compared with the RC group.

Axon regeneration, myelination and activation of Schwann cells. The images of H\&E sections indicated the regenerated nerve fibers in the three groups (Fig. 2A). The number of Schwann cells (blue arrow) detected in the PANI/RC group was greater than that in the RC and sham groups. The axons (black arrow) regenerated more efficiently in the PANI/RC compared with the RC group (Fig. 2A). The blood vessels that were necessary for nutrient supply and neurite growth were detectable in the PANI/RC group. A vast number of fibrous connective tissues (white arrow) were crawled into the nerve that prevented nerve regeneration (Fig. 2A). The protein S100 (marker of Schwann cells) revealed a 3-fold increase in the PANI/RC compared with the sham group (Fig. 2B). The aforementioned protein demonstrated a 1.6-fold increase in the PANI/RC compared with the RC group $(\mathrm{P}<0.01$, Fig. $2 \mathrm{~B}$. The images of toluidine blue sections (Fig. 2B) indicated that the regenerated nerve fibers in the PANI/RC group were smaller and less uniform compared with those in the sham group. However, the nerve fibers in the RC group were the smallest in size and most irregular in shape with numerous fibrous connective tissues. The fiber diameter analysis (Fig. 2D) indicated that the size range in the PANI/RC group was between 3 and $5 \mathrm{~mm}$, whereas the percentage was estimated to $37.6 \pm 5.8$. The latter was similar to the percentage of fiber diameter noted in the sham group (34.2 \pm 6.4$)$ and significantly greater compared with that in the $\mathrm{RC}$ group $(19.2 \pm 6 ; \mathrm{P}<0.01)$.

TEM analysis of the regenerated nerve tissues revealed that the formation of regenerated myelinated fibers occurred at similar levels in both the PANI/RC and sham groups (Fig. 2E). Statistical analysis was carried out on the average axon diameter (Fig. 2D), the thickness of the regenerated myelin sheath (Fig. 2F). A significant difference between the PANI/RC and the $\mathrm{RC}$ groups was noted for all of the parameters measured $(\mathrm{P}<0.05)$. A thicker myelin sheath was observed in the PANI/RC group $(0.93 \pm 0.28 \mu \mathrm{m})$ compared with the $\mathrm{RC}$ group $(0.49 \pm 0.21 \mu \mathrm{m}, \mathrm{P}<0.05)$, yet still smaller than that in sham group $(1.2 \pm 0.27 \mu \mathrm{m} ; \mathrm{P}>0.05)$.

Activation of the ERK1/2/MAPK signaling pathway. Immunohistochemical analysis was employed to analyze the positive expression levels of ERK1/2, 3 months post-implantation, in all groups in order to determine whether the signaling pathways of MAPK/ERK1/2 were activated following the increase in expression of CNTF and BDNF proteins. The positive ratio of MAPK/ERK1/2 proteins in the PANI/RC group was significantly greater than that of the $\mathrm{RC}$ group $(\mathrm{P}<0.01$, Fig. 3A-C). The results further demonstrated that ERK1/2 is activated in the PANI/RC and RC group compared with the sham group (Fig. 3C), as indicated by the increased phosphorylation levels of ERK1/2 (Fig. 3D). The increase in the p-ERK1/2 levels was highly significant between the PANI/RC and the $\mathrm{RC}$ and the sham groups $(\mathrm{P}<0.01)$, while the levels of ERK1/2 were unchanged (Fig. 3D; P>0.05). 

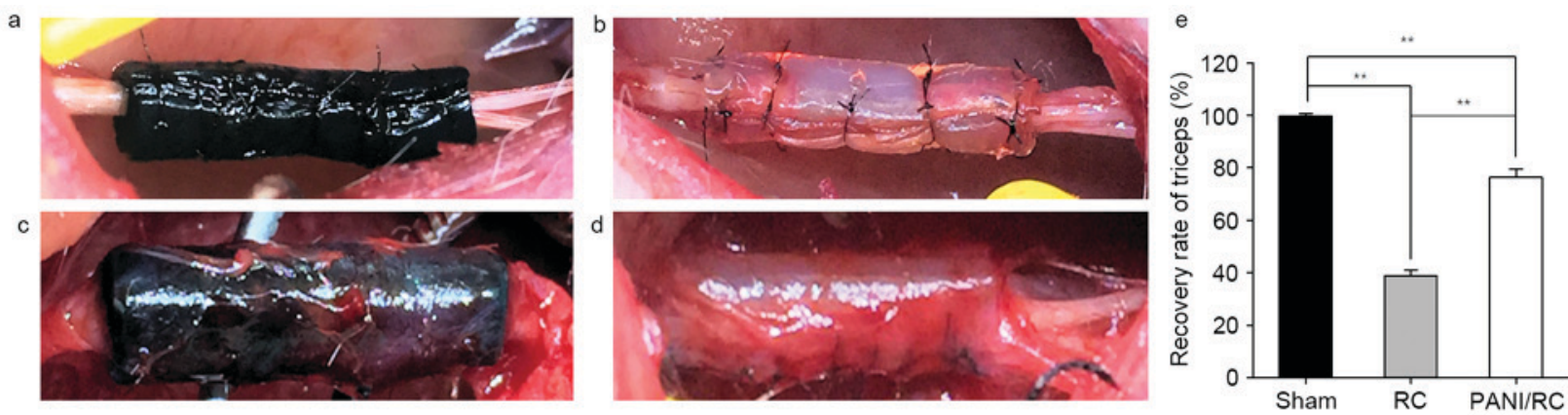

Figure 1. Pre-operative images in the (A) PANI/RC and (B) RC groups and 3-month postoperative images in the (C) PANI/RC and (D) RC groups. (E) Repaired surae comparison between contra lateral operation side with different conduits sham, RC and PANI/RC groups. Data are expressed as the mean \pm standard deviation. $\mathrm{n}=5,{ }^{* * *} \mathrm{P}<0.01$. PANI/RC, polyaniline/regenerated cellulose; $\mathrm{RC}$, regenerated cellulose.
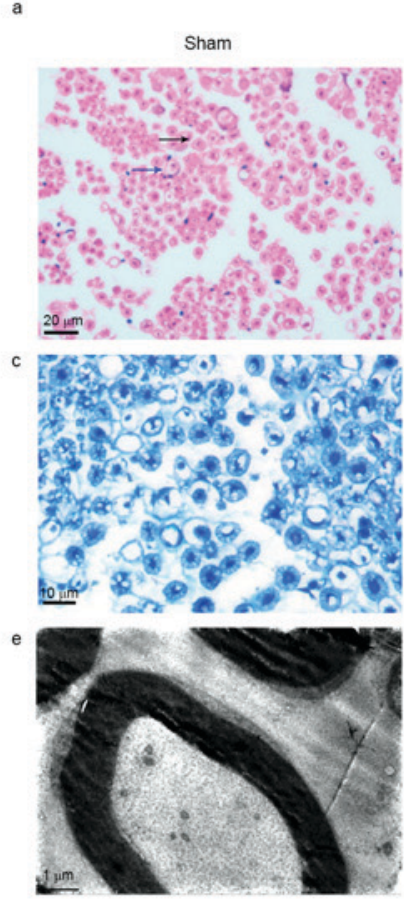

$\mathrm{RC}$
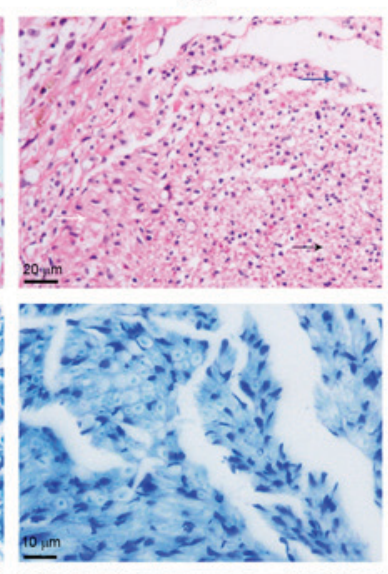

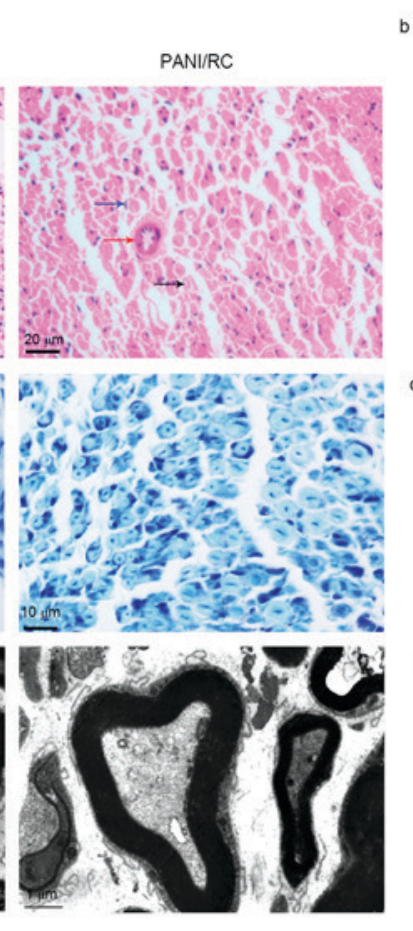

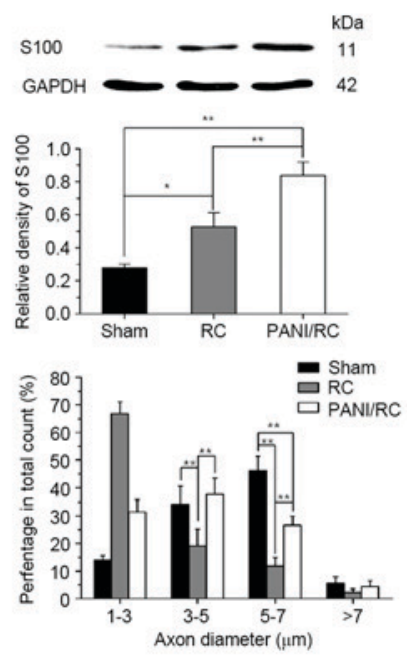

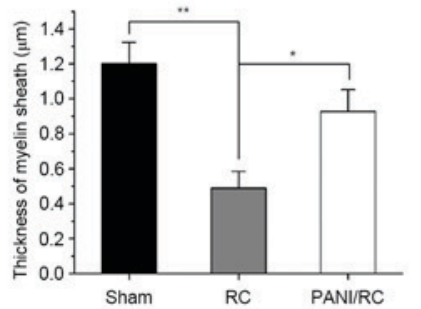

Figure 2. Histology images from cross sections of regenerated nerves extracted from specific types of nerve conduits implanted in rats following 3 months of implantation. Tissues were stained with (A) hematoxylin and eosin, and (B) S100 protein expression levels were measured. (C) Toluidine blue staining. (D) Axon diameter of regenerated and non-operated sciatic nerves. (E) Transmission electron microscopy images. (F) Analysis of the thickness of the myelin sheath. Black arrows indicate the myelinated axons. Blue arrows indicate the Schwann cells. Red arrows indicate the red blood cells/vessels. White arrows indicate the fibrous connective tissue. Data are expressed as the mean \pm standard deviation. $n=5,{ }^{~} \mathrm{P}<0.05,{ }^{* *} \mathrm{P}<0.01$. PANI/RC, polyaniline/cellulose; RC, regenerated cellulose.

Recruitment of growth factors, cytokines and neurotrophins. The expression of the proteins CNTF, BDNF, Tau, GAP-43, NGF and $\alpha$-tubulin following implantation of the conduits was examined using western blot analysis in order to determine the activation of the Schwann cells following nerve injury. The expression levels of the proteins corresponding to the PANI/RC group were significantly higher compared with the expression levels of the proteins corresponding to the sham and RC groups (Fig. 4). Specifically, the expression levels of the proteins BDNF, CNTF, NGF, GAP-43, Tau and $\alpha$-tubulin were significantly increased in the $\mathrm{RC}$ and the PANI/RC groups compared with the sham group $(\mathrm{P}<0.01$, Fig. 4). The proteins
BDNF, CNTF, NGF, GAP-43, Tau and $\alpha$-tubulin revealed a 3.07-, 2.17-, 3.05-, 3.01-, 3.48- and 2.44-fold increase in the PANI/RC group compared with the sham group. The aforementioned proteins demonstrated a 1.27-, 1.18-, 1.43-, 1.59-, 1.64- and 1.24-fold increase in the PANI/RC group compared with the RC group (Fig. 4).

\section{Discussion}

In the present study, the parameters of axons, axon diameter and thickness of myelin sheath were greater in the PANI/RC group compared with the corresponding parameters in the 
$a$

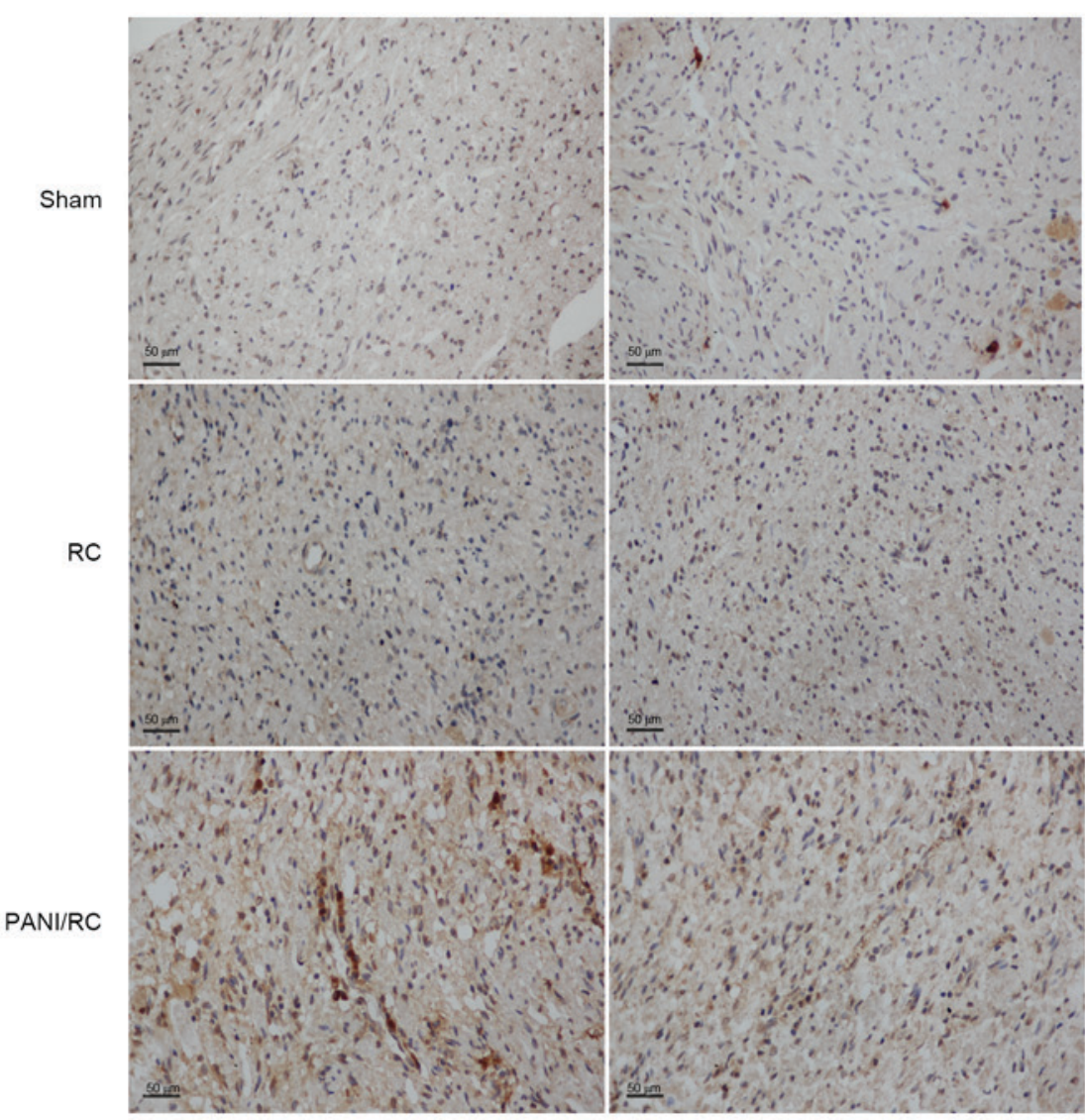

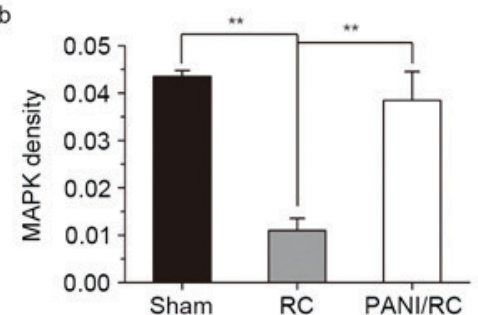

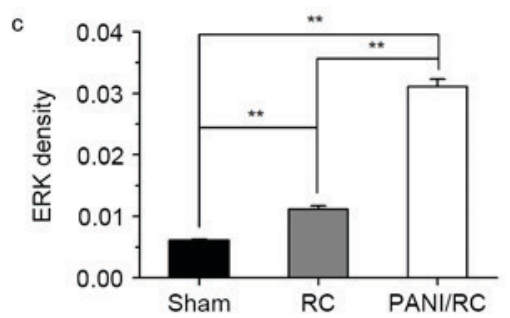

d
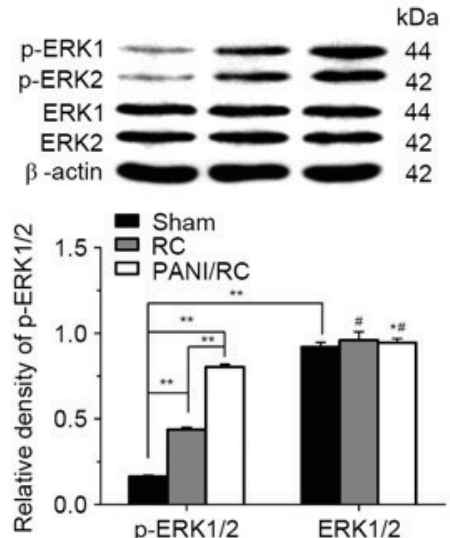

Figure 3. Immunohistochemical images of (A) regenerated nerves and quantification of (B) MAPK and (C) ERK1/2 positive cells. (D) Western blot analysis of p-ERK1/2 in the Sham, RC and PANI/RC groups. Brown staining indicates positive expression. Data are expressed as the mean \pm standard deviation. $\left(\mathrm{n}=5,{ }^{* *} \mathrm{P}<0.01,{ }^{*} \mathrm{P}<0.01\right.$ vs. pERK1/2 in the RC group, ${ }^{* *} \mathrm{P}<0.01$ vs. p-ERK1/2 in the PANI/RC group). PANI/RC, polyaniline/cellulose; ERK, extracellular signal-regulated kinase; MAPK, mitogen-associated protein kinase; $p$, phosphorylated; RC, regenerated cellulose.
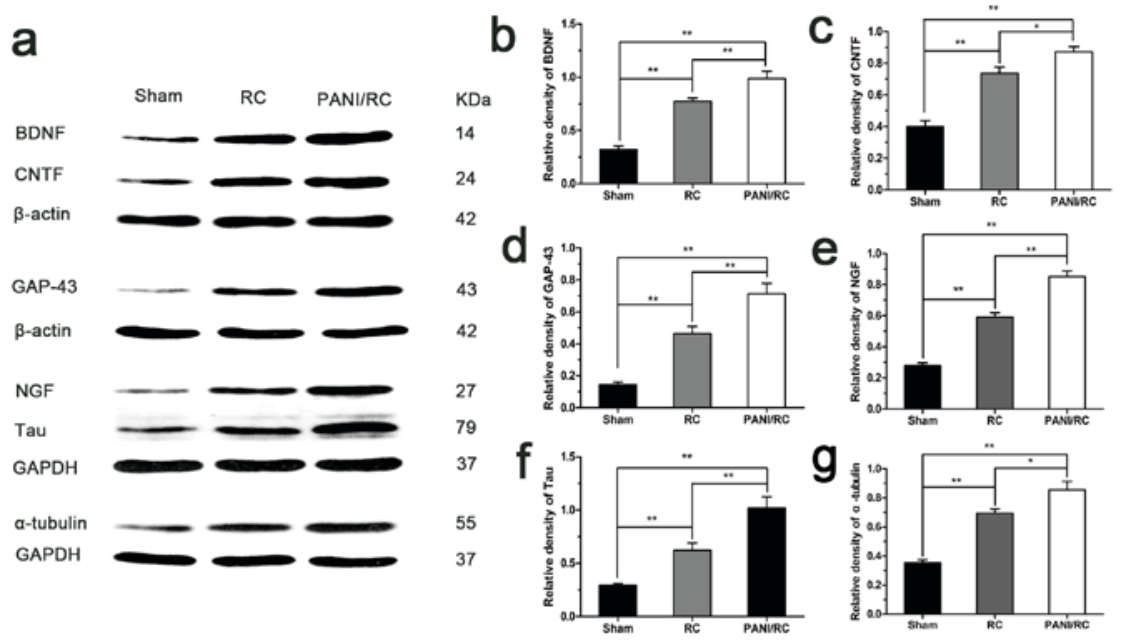

Figure 4. Protein expression levels of BDNF, CNTF, NGF, GAP-43, Tau and $\alpha$-tubulin. (A) Representative western blot images. Protein expression levels of (B) BDNF, (C) CNTF, (D) NGF, (E) GAP-43, (F) Tau and (G) $\alpha$-tubulin, in the three groups. Data are expressed as the mean \pm standard deviation. $n=5$, $\mathrm{P}<0.05$, ${ }^{* *} \mathrm{P}<0.01$. PANI/RC, polyaniline/cellulose; BDNF, brain-derived neurotrophic factor; CNTF, ciliary neurotrophic factor; NGF, nerve growth factor; GAP, growth-associated protein; $\mathrm{RC}$, regenerated cellulose.

$\mathrm{RC}$ group and lower than those noted in the sham group. The histological data were similar with the findings reported in previous studies (17-19).
In the present study, the PANI/RC conduit exhibited two main characteristics: The hierarchical micro-nanostructure and the conductivity (5). The aforementioned factors may 
promote RSC96 cellular proliferation via a synergistic mode of action (5).

Regarding conductivity, some authors have previously reported that the conductive material promotes nerve regeneration even without electrical stimulation (ES) (21-23). The conductive scaffold in the absence of ES can in turn be used to mediate a bioelectricity signal to the distal end for nerve regeneration $(21,22)$. Initial studies in the field of nerve regeneration demonstrated the beneficial effects of a conducting polymer with ES on neurite outgrowth (23). However, an implanted bioelectronic conduit can establish a stable and long-term electrical communication with living tissues, thereby allowing regeneration of the tissues or an exchange of physiological signals in order to recover damaged biological functions (24). The therapeutic actions of ES are mediated by the enhanced BDNF-stimulated myelination that in turn promotes the promyelination effect on Schwann cells at the onset of myelination (25). The results regarding the expression of NGF and BDNF are in agreement with the aforementioned findings. Taken together, the data suggested that the presence of PANI promotes the electrical conductivity of the PANI/RC conduits on axon regeneration and myelination, compared with the RC conduits.

In addition, BDNF activates the MAPK/ERK1/2 cascade, which may lead to transcriptional regulation and protein synthesis in the postsynaptic neuron (26). In the present study, the expression level of BDNF in the PANI/RC group was significantly increased compared with the RC and sham groups. The ERK1/2 (MAPK) signaling pathway of the has demonstrated to be involved in axon regeneration (27). Furthermore, Park et al (28) revealed that CNTF promoted retinal ganglion cell survival and axonal regeneration via the ERK1/2/MAPK signaling pathway, which is similar with the present results.

The aforementioned evidence demonstrated that the release of CNTF and the activation of ERK1/2 were essentially involved in axon growth modulation following nerve injury. Furthermore, the ERK signaling pathway was reported to be involved in the modulation of cytoskeletal protein expression, such as GAP-43 and $\alpha$-tubulin during axon regeneration $(29,30)$. GAP-43 is synthesized at high levels during axonal outgrowth and transported to the growth cone (31). $\alpha$-tubulin participates directly in the regulation of the microtubule polymerization during neuronal development and axonal regeneration (32). Tau is a highly soluble microtubule-associated protein that modulates the stability of axonal microtubules (33). The proteins GAP-43, $\alpha$-tubulin and Tau serve a crucial role in the local cytoskeletal rearrangement at the axonal terminal, causing the growth cone to reform and sprout (34). The level of protein synthesis of GAP-43, Tau and $\alpha$-tubulin during the axon regrowth suggested that these proteins may directly participate in the processes of growth cone formation or axon elongation $(30,32,34)$. In the present study, western blot analysis was carried out to determine whether BDNF, CNTF, GAP-43, Tau and/or $\alpha$-tubulin proteins were activated during axon regeneration, so as to establish a link between cytokine signaling and axon regeneration. The present study indicated that the expression of the GAP-43, $\alpha$-tubulin and Tau proteins in the PANI/RC group was elevated compared with the expression of the corresponding proteins in the sham and RC groups.
These findings are in agreement with previous studies that investigated the expression levels of GAP-43 and $\alpha$-tubulin ( $\sim$ 10-fold and $\sim 3$-5-fold increase, respectively) (35).

In the present study, the synthetic PANI/RC conduit exhibited favorable efficacy for nerve tissue regeneration. The aforementioned PANI conduit enhanced the sciatic nerve elongation and provided a favorable environment for axon regeneration by promoting the secretion of numerous cytokines, growth factors and neurotrophins, as opposed to the RC conduit. Taken together, the increasing levels of the CNTF and BDNF proteins induced by PANI regulated the activation of the ERK1/2 (MAPK) signaling pathway that in turn promoted the expression of the GAP-43, tau and $\alpha$-tubulin cytoskeletal proteins.

In conclusion, the findings of the present study suggested that in the presence of PANI, the PANI/RC conduit serves a pivotal role in the stimulation of Schwann cells, in the activation of the ERK1/2 (MAPK) signaling pathway and in the release of significant growth factors that are required for the enhancement of axon regeneration and myelination following static nerve injury. Despite the promising findings, additional research is required to elucidate the exact mechanism(s) of the conduit action and which characteristic is more important for nerve regeneration: The hierarchical micro-nanostructure or the conductivity.

\section{Acknowledgements}

The present study was supported by the National Natural Science Foundation of China: United Foundation with Xinjiang (grant no. U1403222).

\section{References}

1. Siemionow M and Brzezicki G: Chapter 8: Current techniques and concepts in peripheral nerve repair. Int Rev Neurobiol 87: 141-172, 2009.

2. Buchthal F and Kühl V: Nerve conduction, tactile sensibility, and the electromyogram after suture or compression of peripheral nerve: A longitudinal study in man. J Neurol Neurosurg Psychiatry 42: 436-451, 1979.

3. Evans GR: Peripheral nerve injury: A review and approach to tissue engineered constructs. Anat Rec 263: 396-404, 2001.

4. Burnett MG and Zager EL: Pathophysiology of peripheral nerve injury: A brief review. Neurosurg Focus 16: E1, 2004.

5. Xu D, Fan L, Gao L, Xiong Y, Wang Y, Ye Q, Yu A, Dai H, Yin Y, Cai J and Zhang L: Micro-nanostructured polyaniline assembled in cellulose matrix via interfacial polymerization for applications in nerve regeneration. ACS Appl Mater Interfaces 8: 17090-17097, 2016.

6. Bhatheja K and Field J: Schwann cells: Origins and role in axonal maintenance and regeneration. Int J Biochem Cell Biol 38: 1995-1999, 2006.

7. Mar FM, Bonni A and Sousa MM: Cell intrinsic control of axon regeneration. EMBO Rep 15: 254-263, 2014.

8. Makwana M and Raivich G: Molecular mechanisms in successful peripheral regeneration. FEBS J 272: 2628-2638, 2005.

9. Benraiss A, Chmielnicki E, Lerner K, Roh D and Goldman SA: Adenoviral brain-derived neurotrophic factor induces both neostriatal and olfactory neuronal recruitment from endogenous progenitor cells in the adult forebrain. J Neurosci 21: 6718-6731, 2001.

10. Acheson A, Conover JC, Fandl JP, DeChiara TM, Russell M, Thadani A, Squinto SP, Yancopoulos GD and Lindsay RM: A BDNF autocrine loop in adult sensory neurons prevents cell death. Nature 374: 450-453, 1995.

11. Huang EJ and Reichardt LF: Neurotrophins: Roles in neuronal development and function. Annu Rev Neurosci 24: 677-736, 2001. 
12. Tom VJ, Sandrow-Feinberg HR, Miller K, Domitrovich C, Bouyer J, Zhukareva V, Klaw MC, Lemay MA and Houlé JD: Exogenous BDNF enhances the integration of chronically injured axons that regenerate through a peripheral nerve grafted into a chondroitinase-treated spinal cord injury site. Exp Neurol 239: 91-100, 2013

13. Friedman B, Scherer SS, Rudge JS, Helgren M, Morrisey D, McClain J, Wang DY, Wiegand SJ, Furth ME, Lindsay RM, et al: Regulation of ciliary neurotrophic factor expression in myelin-related Schwann cells in vivo. Neuron 9: 295-305, 1992.

14. Zhang J, Lineaweaver WC, Oswald T, Chen Z, Chen Z and Zhang F: Ciliary neurotrophic factor for acceleration of peripheral nerve regeneration: An experimental study. J Reconstr Microsurg 20: 323-327, 2004.

15. Cen LP, Luo JM, Zhang CW, Fan YM, Song Y, So KF, van Rooijen N, Pang CP, Lam DS and Cui Q: Chemotactic effect of ciliary neurotrophic factor on macrophages in retinal ganglion cell survival and axonal regeneration. Invest Ophthalmol Vis Sci 48: 4257-4266, 2007.

16. Zhang CW, Lu Q, You SW, Zhi Y, Yip HK, Wu W, So KF and Cui Q: CNTF and BDNF have similar effects on retinal ganglion cell survival but differential effects on nitric oxide synthase expression soon after optic nerve injury. Invest Ophthalmol Vis Sci 46: 1497-1503, 2005.

17. Hu Y, Leaver SG, Plant GW, Hendriks WT, Niclou SP Verhaagen J, Harvey AR and Cui Q: Lentiviral- mediated transfer of CNTF to schwann cells within reconstructed peripheral nerve grafts enhances adult retinal ganglion cell survival and axonal regeneration. Mol Ther 11: 906-915, 2005.

18. Lin KL, Yang DY, Chu IM, Cheng FC, Chen CJ, Ho SP and Pan HC: DuraSeal as a ligature in the anastomosis of rat sciatic nerve gap injury. J Surg Res 161: 101-110, 2010.

19. Oliveira EF, Mazzer N, Barbieri CH and DelBel EA: The use of a muscle graft to repair a segmentary nerve defect. An experimental study using the sciatic nerve of rats as model. J Neurosci Methods 133: 19-26, 2004

20. Weber RA, Warner MR, Verheyden $\mathrm{CN}$ and Proctor WH: Functional evaluation of gap vs. abutment repair of peripheral nerves in the rat. J Reconstr Microsurg 12: 159-163, 1996.

21. Lee JY, Bashur CA, Goldstein AS and Schmidt CE: Polypyrrole-coated electrospun PLGA nanofibers for neural tissue applications. Biomaterials 30: 4325-4335, 2009.

22. Huang J, Lu L, Zhang J, Hu X, Zhang Y, Liang W, Wu S and Luo Z: Electrical stimulation to conductive scaffold promotes axonal regeneration and remyelination in a rat model of large nerve defect. PLoS One 7: e39526, 2012.
23. Schmidt CE, Shastri VR, Vacanti JP and Langer R: Stimulation of neurite outgrowth using an electrically conducting polymer. Proc Natl Acad Sci USA 94: 8948-8953, 1997.

24. Zhu B, Luo SC, Zhao H, Lin HA, Sekine J, Nakao A, Chen C, Yamashita Y and Yu HH: Large enhancement in neurite outgrowth on a cell membrane-mimicking conducting polymer. Nat Commun 5: 4523, 2014.

25. Huang J, Hu X, Lu L, Ye Z, Zhang Q and Luo Z: Electrical regulation of Schwann cells using conductive polypyrrole/chitosan polymers. J Biomed Mater Res A 93: 164-174, 2010.

26. Mohajerani MH, Sivakumaran S, Zacchi P, Aguilera P and Cherubini E: Correlated network activity enhances synaptic efficacy via BDNF and the ERK pathway at immature CA3 CA1 connections in the hippocampus. Proc Natl Acad Sci USA 104 13176-13181, 2007.

27. Abe $\mathrm{N}$ and Cavalli V: Nerve injury signaling. Curr Opin Neurobiol 18: 276-283, 2008

28. Park K, Luo JM, Hisheh S, Harvey AR and Cui Q: Cellular mechanisms associated with spontaneous and ciliary neurotrophic factor cAMP-induced survival and axonal regeneration of adult retinal ganglion cells. J Neurosci 24: 10806-10815, 2004.

29. Chen ZL, Yu WM and Strickland S: Peripheral regeneration. Annu Rev Neurosci 30: 209-233, 2007.

30. Kaneda M, Nagashima M, Mawatari K, Nunome T, Muramoto K, Sugitani $\mathrm{K}$ and Kato S: Growth-associated protein43 (GAP43) is a biochemical marker for the whole period of fish optic nerve regeneration. Adv Exp Med Biol 664: 97-104, 2010.

31. Janke C and Bulinski JC: Post-translational regulation of the microtubule cytoskeleton: Mechanisms and functions. Nat Rev Mol Cell Biol 12: 773-786, 2011.

32. Xu MF, Zhou H, Hu CY, Liang YQ, Hu L and Chen D: The mechanisms of EGFR in the regulation of axon regeneration. Cell Biochem Funct 32: 101-105, 2014.

33. Liu K, Tedeschi A, Park KK and He Z: Neuronal intrinsic mechanisms of axon regeneration. Annu Rev Neurosci 34: 131-152, 2011.

34. Skene JH, Jacobson RD, Snipes GJ, McGuire CB, Norden JJ and Freeman JA: A protein induced during nerve growth (GAP-43) is a major component of growth-cone membranes. Science 233: 783-786, 1986.

35. Fernandes KJL, Fan DP, Tsui BJ, Cassar SL and Tetzlaff W: Influence of the axotomy to cell body distance in rat rubrospinal and spinal motoneurons: Differential regulation of GAP-43, tubulins, and neurofilament-M. J Comp Neurol 414: 495-510, 1999. 